\title{
Translational dynamics of a cold water cluster in the presence of an external uniform electric field
}

\author{
Alice Vegiria) \\ National Hellenic Research Foundation, Institute of Theoretical and Physical Chemistry, \\ 48 Vas. Constantinou Ave., Athens, 11635 Greece
}

(Received 2 January 2002; accepted 5 March 2002)

\begin{abstract}
Molecular dynamics simulations for a TIP4P water cluster consisting of 32 molecules at $T$ $=200 \mathrm{~K}$, under the influence of a broad range of constant electric fields $\left(0.5-7.0 \times 10^{7} \mathrm{~V} / \mathrm{cm}\right)$, are presented. This work focuses on the evolution of the single particle translational dynamics, mainly along the field axis as the field is progressively increased, by means of mean-square-displacement curves, the self-part of the van Hove distribution functions and the intermediate scattering functions. Two critical fields have been identified, the one, $\left(E_{C 1}=1.5 \times 10^{7} \mathrm{~V} / \mathrm{cm}\right)$ assigned to the onset of the dipole alignment and the second one $\left(E_{C 2}=5.0 \times 10^{7} \mathrm{~V} / \mathrm{cm}\right)$ to the onset of crystallization. These transitions are marked by an abrupt increase of the corresponding structure relaxation times, which remain nearly constant for electric fields between $E_{C 1}$ and $E_{C 2}$. Structure relaxation has been found to obey stretched exponential dynamics, whereas the $Q$ dependence of the relaxation times, for all fields, followed a power law. Fields weaker than $E_{C 1}$ have been found to induce a weakening of the molecular interactions. In this case, the system develops a dynamic behavior similar to that met in the liquid. (C) 2002 American Institute of Physics. [DOI: 10.1063/1.1473657]
\end{abstract}

\section{INTRODUCTION}

The process of producing solid structures that possess long-range translational and orientational order through the application of a uniform electric field is known as electrofreezing. Similarly, the application of a constant magnetic field $^{1}$ to a system of magnetic chains can result in their alignment along the field lines and to the solidification of the system under strong enough magnetic fields. Although the development of orientational order is easy to understand because of the tendency of the electric or magnetic dipoles to align along the field lines, the development of translational order is not so obvious and is the result of coupling between the orientational and translational motions.

Recently, it was possible to produce ice cubic structures in molecular dynamics (MD) simulations ${ }^{2-13}$ by applying a rather strong electric field on supercooled liquid water. Although this transformation in bulk water and in Stockmayer fluids ${ }^{14,15}$ has been studied in some detail, mainly from the structural point of view, the precise route to crystallization with increasing field strength and the concomitant change in dynamics has not been given equal attention.

In a series of previous publications ${ }^{16,17}$ we chose to study a moderate in size water cluster instead, mainly for computational reasons, (low cost computational effort and avoidance of boundary conditions which might impose unnecessary effects). The conclusions drawn from this work cannot be safely applied directly to the bulk, but they can serve as a guideline about what one could expect to happen there. By analyzing structural transformations with increasing electric field, we found that the system at a temperature

\footnotetext{
a) Author to whom correspondence should be addressed; electronic mail: avegiri@eie.gr
}

of about $200 \mathrm{~K}$, before crystallizing passes through an intermediate phase, which consists of a number of stacked cubes. ${ }^{17}$ This phase survives only within a limited range of electric fields. The number of molecules participating in well-defined nanotubes was found to increase with temperature lowering. This transition, which is associated with the sudden flipping of the molecular dipoles from random directions to directions restricted within a solid angle between 0 and $\pi / 2$ with respect to the field, entails the characteristics of a first-order phase transition and is taking place at a critical field, which for the system under examination was found equal to $E_{C}=1.5 \times 10^{7} \mathrm{~V} / \mathrm{cm}$. Based exclusively on the analysis of structures, we were not able to identify any discontinuities that would signal a transition to the crystalline phase. However, we could identify an intermediate region, between that of the stacked cubes and the crystalline phase, where the system did not seem to display any of the characteristics of either the nanotube or the crystalline phase.

On the other hand, by examining the reorientational motions of the intrinsic molecular axes ${ }^{17}$ we found a substantial increase in the axes reorientational rates with the field, as compared to the corresponding rates in the absence of the field. Additionally, the relaxation of the axes was found to follow stretched exponential dynamics. This kind of behavior was interpreted as an indication of the loosening of the molecular interactions with the application of the field. However, in the strong field regime, the reorientational rates were found to decrease.

In the present work we extend our MD calculations to longer times, from 1.5 to $2.5 \mathrm{~ns}$ depending on the field strength, aiming to the investigation of the single particle transport as a function of the external electric field and the dynamics of the structural transformations at directions par- 
allel to the field lines. Our main finding is that there are two transitional electric fields. The first one, denoted as $E_{C 1}$, is associated with the dipole flipping transition and is equal to $E_{C}$ as found previously, ${ }^{16,17}$ and the second one, denoted as $E_{C 2}$, is associated with the transition to the crystalline phase. For both $E_{C 1}$ and $E_{C 2}$ structural relaxation times showed an abrupt increase. In addition, the loosening of the immediate molecular environment for relatively weak fields, i.e., for 0 $<E<E_{C 1}$, was unambiguously verified, through the substantial decrease of the structure relaxation times.

That the water cluster can pass through those two different transition points as the field is increased, is something that has been observed before in electrorheological (ER) fluids when subjected to an external electric field at a fixed temperature. ${ }^{18,19}$ An ER fluid consists of a suspension of dielectric particles in a liquid of low dielectric constant, interacting through induced dipole-induced dipole forces. For electric fields below a critical value, the system is found in a fluid state. In an intermediate region it forms chains along the field direction, but randomly distributed in a perpendicular plane, whereas for fields stronger than a second critical value, the system solidifies to a tetragonal lattice.

In computer simulations of water, the crystal state is not possible to be obtained by simply cooling down the system, because of the much larger cooling rates used than in a real life experiment. Instead, the system reaches the glassy state, which has been extensively studied, both theoretically ${ }^{20-25}$ and experimentally. ${ }^{26-28}$ Since the application of an external electric field acts macroscopically along the same direction as the lowering of temperature, one is challenged to pose questions about the nature of the states that are obtained, at least for certain fields, about whether these states can be identified with the glassy state, and also, about whether concepts and dynamic laws developed by the mode coupling theory $(\mathrm{MCT})^{29}$ for the description of the glassy state can be applicable here as well.

In this paper, we briefly review the model and the computational procedure, which is the same as in our previous work, ${ }^{16,17}$ (Sec. II). Next, in Sec. III, which is divided into three subsections, we present and discuss the results from the calculation of the mean-square displacement of the center of mass, the self-part of the van Hove particle density correlation functions, and the intermediate scattering functions. A summary of the main findings and conclusions follows in Sec. IV.

\section{METHOD OF CALCULATION}

We have carried out constant energy molecular dynamics (MD) simulations for a relatively small water cluster $(N$ $=32$ ) at $T=200 \mathrm{~K}$, under several external electric fields in the $0-7 \times 10^{7} \mathrm{~V} / \mathrm{cm}$ range. Computational details are found in Ref. 17. The difference with previous calculations is that in the present case the trajectories are integrated to longer times $\left(\sim 2.5 \mathrm{~ns}\right.$ for $E=7 \times 10^{7} \mathrm{~V} / \mathrm{cm}$ and $\sim 1.5 \mathrm{~ns}$ for the rest), because of the slow relaxation of the translational motions at stronger fields.

The total energy of the system consists of the following terms:
TABLE I. Temperature of the cluster as a function of the external field.

\begin{tabular}{cc}
\hline \hline$E\left(10^{7} \mathrm{~V} / \mathrm{cm}\right)$ & $T(\mathrm{~K})$ \\
\hline 0 & 202 \\
0.5 & 197 \\
1 & 204 \\
1.5 & 195 \\
2 & 204 \\
3 & 204 \\
4 & 204 \\
5 & 197 \\
7 & 197 \\
\hline \hline
\end{tabular}

$$
U=U_{\text {pair }}^{\mathrm{w}-\mathrm{w}}+U^{\mathrm{w}-\text { field }}+U_{\mathrm{pol}}^{\mathrm{w}-\text { field }}+U_{\text {ind }}^{\mathrm{w}-\mathrm{w}} .
$$

$U_{\text {pair }}^{\mathrm{w}-\mathrm{w}}$ accounts for the pairwise water-water interactions. For this purpose the TIP4P ${ }^{30}$ potential model has been employed.

$U^{\mathrm{w}-\text { field }}$ is the interaction energy between the permanent charges of water molecules and the external field and is equal to

$$
U^{\mathrm{w}-\text { field }}=-\sum_{i=1}^{N} \sum_{k=1}^{3} q_{k}\left(\mathbf{r}_{k}^{i} \cdot \mathbf{E}\right),
$$

$\mathbf{r}_{k}^{i}$ is the position vector of the $k^{\text {th }}$ point charge of the $i^{\text {th }}$ molecule and $E$ is the external field directed along the $z$ axis.

$U_{\text {pol }}^{\mathrm{w}-\text { field }}$ is the polarization energy of water molecules under a uniform external field $E$. It is given as

$$
U_{\text {pol }}^{\mathrm{w}-\text { field }}=-\alpha_{w} \frac{E^{2}}{2},
$$

where $a_{w}=1.44 \AA^{3}$ is the isotropic polarizability of an isolated water molecule.

$U_{\text {ind }}^{\mathrm{w}-\mathrm{w}}$ describes the induced dipole-permanent charge interactions between any two water molecules.

$$
U_{\text {ind }}^{\mathrm{w}-\mathrm{w}}=-\sum_{i, j} \mathbf{E}_{i j}^{(1)} \cdot \mathbf{p}_{j}^{\text {ind }}
$$

where $E_{i j}^{(1)}$ is the electric field produced by the permanent charge $q_{k}$ of the $i^{\text {th }}$ water molecule at the oxygen atom of the $j^{\text {th }}$ molecule

$$
E_{i j}^{(1)}=\sum_{k=1}^{3} \frac{q_{k}}{\left|\mathbf{r}_{0}^{j}-\mathbf{r}_{k}^{i}\right|^{3}} \cdot\left(\mathbf{r}_{0}^{j}-\mathbf{r}_{k}^{i}\right),
$$

where $\mathbf{r}_{0}^{j}$ is the position vector of the oxygen atom of the $j^{\text {th }}$ molecule

$$
\mathbf{p}_{i}^{\text {ind }}=\alpha_{w} \mathbf{E}
$$

is the dipole moment of the $i^{\text {th }}$ molecule induced by the external electric field $E$.

In this calculation induced dipole-induced dipole interaction terms have been omitted. An explanation can be found in Ref. 17.

Temperature is determined from the average kinetic energy of the cluster and for all fields it was found to fluctuate evenly about a mean value, indicating cluster equilibration. Temperatures are listed in Table I. 


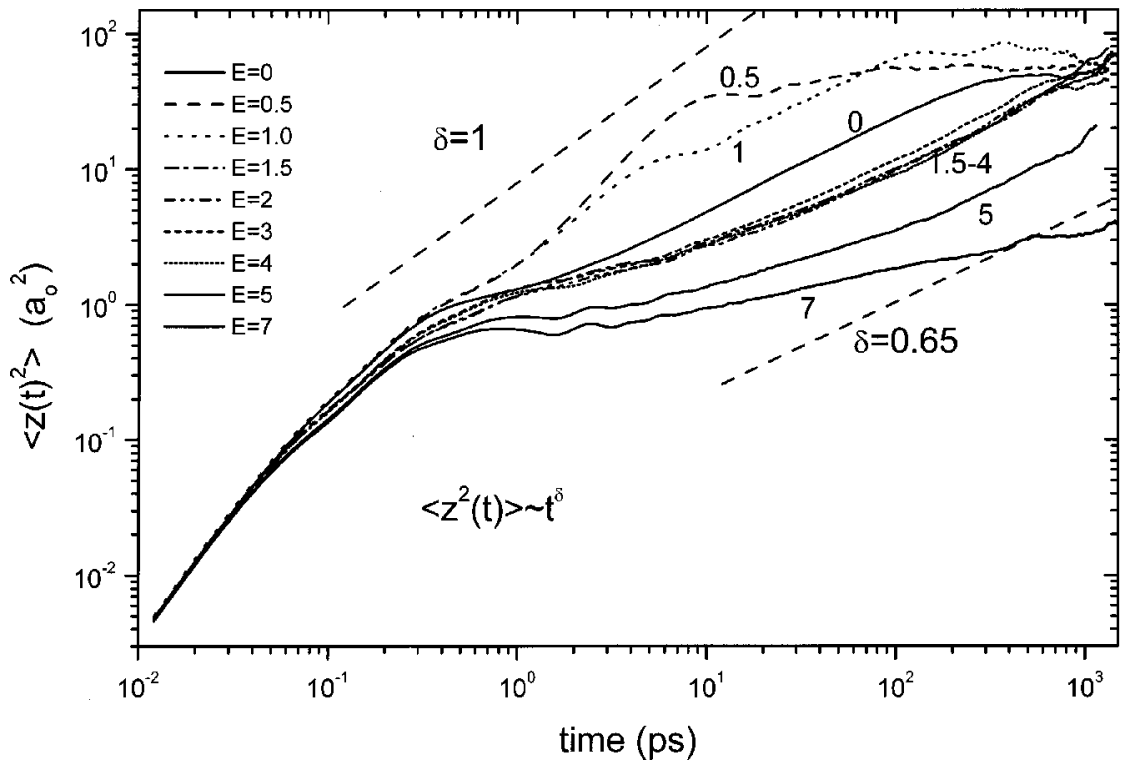

FIG. 1. Mean-square-displacement curves along the $z$ axis. Electric field values, in $10^{7} \mathrm{~V} / \mathrm{cm}$ are displayed both in the inset and on the curves.
We should like to note that in the water cluster-electric field system, total angular momentum is not a constant of motion, except from the $L_{z}$ component, which is conserved. Since at $t=0$ the cluster is given zero total angular momentum, (by assuming zero angular and translational velocities of the molecules), $L_{z}$ remains almost zero throughout the calculation, but not the $L_{x}$ and the $L_{y}$ components whose time variation depends on the time variation of the projection of the total dipole moment of the cluster on the $y$ and $x$ space-fixed axes, respectively,

$$
\begin{aligned}
& \frac{d L_{x}}{d t}=M_{y} E, \\
& \frac{d L_{y}}{d t}=-M_{x} E, \\
& \frac{d L_{z}}{d t}=0 .
\end{aligned}
$$

The implications of a nonzero and nonconserved total angular momentum are that the translational and rotational motions of the individual water molecules are modulated by low frequency components, in the $3-10 \mathrm{~cm}^{-1}$ range, depending on the field strength. However, the way the total angular momentum interferes with the relaxation of the structure and the particle transport is an issue that has its own merit and it should be investigated separately in more detail.

\section{RESULTS AND DISCUSSION}

\section{A. Mean-square-displacements along field lines (MSD_z)}

The MSD- $z$ curves $\left\langle z(t)^{2}\right\rangle$, of the single particle coordinates along the field axis, for fields between $E=0$ and $E$ $=7 \times 10^{7} \mathrm{~V} / \mathrm{cm}$ are displayed in Fig. 1 on a double logarithmic plot. Here $z$ is the coordinate of the oxygen atom. Since the molecular system we are dealing with is finite, $\left\langle z(t)^{2}\right\rangle$ is bounded by $R_{S}{ }^{2}(E)$, where $R_{S}$ is defined as the distance from the one end of the cluster to the other along the $z$ axis.
$R_{S}$ depends on the external field, because of the corresponding change of shape that is imposed on the cluster. ${ }^{16,17}$ Thus for $E=0, R_{S}$ is about 23 ao, whereas for fields larger than the critical field $E_{C 1}=1.5 \times 10^{7} \mathrm{~V} / \mathrm{cm}, R_{S}$ is about 60 ao. ${ }^{17}$ The flattening of the curves we observe at the long time limit for fields less than $E_{C 1}$ corresponds to particle diffusion to distances less than the position of their next-nearest neighbors along the $z$ axis. Events that a particle can diffuse to larger distances do occur but they are rare. The fact that the asymptote of $\left\langle z(t)^{2}\right\rangle$ is reached faster for $E=0.5 \times 10^{7}$ and $E$ $=1.0 \times 10^{7} \mathrm{~V} / \mathrm{cm}$ than for any other field, implies a larger particle mobility.

If we take a closer look at the MSD_z curve that corresponds to $E=0$ we observe the appearance of a plateau which extends from about 0.4 up to $1 \mathrm{ps}$. According to MCT, plateau in the $\log -\log$ plot of the MSD curves versus time, signify the existence of the "caging effect," a concept introduced to explain the nature of the diffusive motions in supercooled liquids and glasses. Thus, particles appear to be trapped into transient cages formed by their immediate neighbors for a considerable amount of time, before they start diffusing away after the cage is relaxed. The plateau is the result of rattling, nondiffusive motions of the particles inside their cages. The more restricted the motion is, the more pronounced the plateau becomes, extending, sometimes into several decades of time, as is the case in supercooled water. ${ }^{22}$ In the present case the cluster is found either in the solid-like or in the solid-liquid coexistence regime, as is the case for clusters of this size and temperature $(\sim 200 \mathrm{~K})$. Therefore, a significant degree of trapping is expected at $E$ $=0$. However, with the switching on of the field, at $E=0.5$ $\times 10^{7}$ and $E=1.0 \times 10^{7} \mathrm{~V} / \mathrm{cm}$, the plateau disappears, as would happen if the temperature were raised. The plateau reappears for $E \geqslant E_{C 1}$ and it becomes quite pronounced for $E \geqslant 5.0 \times 10^{7} \mathrm{~V} / \mathrm{cm}$ when the field-induced cluster crystallization is initiated. ${ }^{17}$

In cases of normal transport, (simple Brownian motion), self-diffusion constants $(D)$ are calculated from the long-time 
slope of the corresponding MSD curves, according to the Einstein relation, which in one dimension reads as $\left\langle z^{2}(t)\right\rangle$ $=2 D t$. However, in cases of anomalous transport, the mean square displacement follows an algebraic dependence on time

$$
\left\langle z^{2}(t)\right\rangle \propto t^{\delta},
$$

where $\delta=1$ corresponds to the Brownian limit. Whenever $\delta>1$ or $\delta<1$ the system is found in the enhanced or subdiffusion (dispersive) regime, respectively. In anomalous diffusion, $D$ depends on time and cannot be defined rigorously. Then

$$
D(t) \approx \frac{d}{d t}\left\langle r^{2}(t)\right\rangle \approx t^{\delta-1}
$$

and if $\delta<1 D(t)$ tends to zero on longer time scales. Instead, one can introduce a generalized diffusion coefficient, $K_{\delta},{ }^{31}$ which has a dimension of $\mathrm{cm}^{2} \mathrm{~s}^{-\delta}$ and is defined as

$$
\left\langle z_{t \rightarrow \infty}^{2}(t)\right\rangle=\frac{2 K_{\delta}}{\Gamma(1+\delta)} t^{\delta} .
$$

The upper dash line in Fig. 1 is drawn with a slope equal to 1 and by comparing it with the slopes of the MSD_z curves we immediately see that the cluster is in the dispersive regime for all but the $E=0.5 \times 10^{7} \mathrm{~V} / \mathrm{cm}$ electric field, where in this case $\delta$ is close to 1 . The slopes for all other fields, taken from the time interval between 10 and 100 ps for $E<E_{C 1}$, and between 100 and 800 ps for $E_{C 1} \leqslant E \leqslant 0.5$ $\times 10^{7} \mathrm{~V} / \mathrm{cm}$, are almost identical and equal to $\sim 0.65$, (compare with the slope of the lower dash line). It is remarkable that all MSD_z curves for $E_{C 1} \leqslant E \leqslant 4.0 \times 10^{7} \mathrm{~V} / \mathrm{cm}$ coalesce into a single curve. This is not accidental, as we shall see later in Sec. III C, since the system for this range of fields displays similar dynamic behavior. For $E=7.0 \times 10^{7} \mathrm{~V} / \mathrm{cm}$ the slope is quite small and the particles, on the average, do not abandon their immediate environment. In the time window of our calculations no transition to normal transport has been observed. Specifically for $E=0$, we extended the calculation to 3 ns. However, even for such a long time, we were not able to observe a change of slope in MSD at longer times. Finally, at very short times, the motion is ballistic with the MSD- $z$ curves displaying a slope of about 1.9.

Subdiffusion is related to some sort of retarded motion and can arise from either random walks in fractal structures, (spatial disorder) or from nonexponential waiting time distributions, (temporal disorder). ${ }^{32}$ In the latter case, waiting time distributions possess long time tails, of the form $\psi(t)$ $\approx t^{-1-\delta}$, for which an average characteristic time cannot be defined. In fact, $\langle t\rangle=\infty$. In this case, subdiffusion is attributed to the existence of traps, the realization of which are the molecular cages, and to rare hopping events between them. The origin of subdiffusion is currently under investigation and the results will be presented in a separate publication. Certainly subdiffusion is not related to the finiteness of the system, since MSD calculated at a higher temperature, ( $T$ $\sim 290 \mathrm{~K}$ ), displayed a linear time dependence at longer times.
The characteristic clustering of the MSD_z curves in a certain field region gives the impression that the dynamic behavior of the system does not change uniformly with the field, and that there must be regions where the system displays similar dynamics. One may infer, that the cluster is in one state for $0<E<E_{C 1}$, in another one for $E_{C 1} \leqslant E \leqslant 4.0$ $\times 10^{7} \mathrm{~V} / \mathrm{cm}$, and in a completely different one for $E>4.0$ $\times 10^{7} \mathrm{~V} / \mathrm{cm}$. From our previous work, ${ }^{16,17}$ based on the examination of structural characteristics and reorientational rates of the molecular intrinsic axes, we already know, that the system turns eventually to a crystal for strong fields and that there must be a loosening of the molecular interactions for weaker fields. We had identified a transition field $E_{C 1}$, which was associated with the flipping of the molecular dipole moment vectors, but we had failed to identify a second sharp transition as the system proceeded to crystallization. The picture we get from the MSD_z curves directs to the existence of a second transition field $E_{C 2}$, at 5 $\times 10^{7} \mathrm{~V} / \mathrm{cm}$. Our further analysis strengthens this observation.

In Figs. 2(a)-2(d) we plot typical single particle trajectories for $E=0,0.5 \times 10^{7}, 1 \times 10^{7}$, and $4 \times 10^{7} \mathrm{~V} / \mathrm{cm}$, projected onto the $x z$ plane. The $x$ and $y$ directions, which are normal to the field, are identical. For $E=0$ sticking areas, assigned to restricted motion, inside cages are clearly observed, a fact which is consistent with the sublinear behavior with time of the corresponding MSD_z curve and the existence of the plateau. Thus for this temperature a distinction between local vibrations and translational motions can be made. Such sticking areas are also observed for $E=1$ $\times 10^{7} \mathrm{~V} / \mathrm{cm}$, however, in a lesser degree. The difference, however, is for $E=0.5 \times 10^{7} \mathrm{~V} / \mathrm{cm}$, where the trajectory can be characterized as ergodic, since it fills the entire available space. No restriction in motion is observed, as is for the Brownian case. Here local vibrational and translational motions cannot be distinguished anymore and this is signified by the disappearance of the plateau in the corresponding MSD_ $z$ curve. For fields $E \geqslant E_{C 1}$ the situation changes dramatically. The molecules become localized in the $x-y$ plane from where excursions to adjacent planes along the $z$ axis are in terms of jump motions. Notice that a molecule is free to explore the $x y$ plane, because of the absence of any lateral hydrogen bonds, as shown previously in Ref. 17. For these fields, all dipoles have flipped along the direction of the field and because of their mutual repulsion they stay apart at distances of about 7 ao.

For all fields $E \geqslant E_{C 1}$ three characteristic types of trajectories have been identified; those that remain confined within the $x-y$ plane for the entire simulation time, those that perform a single vertical (along the $z$ axis) jump to first neighbors, and those that perform double vertical jumps up to second neighbors. These representative trajectories, projected onto the $x-z$ plane, are displayed in Figs. 3(a)-3(d). However, as fields become even stronger, $(E>5$ $\left.\times 10^{7} \mathrm{~V} / \mathrm{cm}\right)$, there are trajectories that become arrested at specific sites even in the $x-y$ plane, an indication of system crystallization. Such a trajectory for $E=7 \times 10^{7} \mathrm{~V} / \mathrm{cm}$ is illustrated in frame (d) of Fig. 3. Notice, that the axes in all frames are of the same length. 

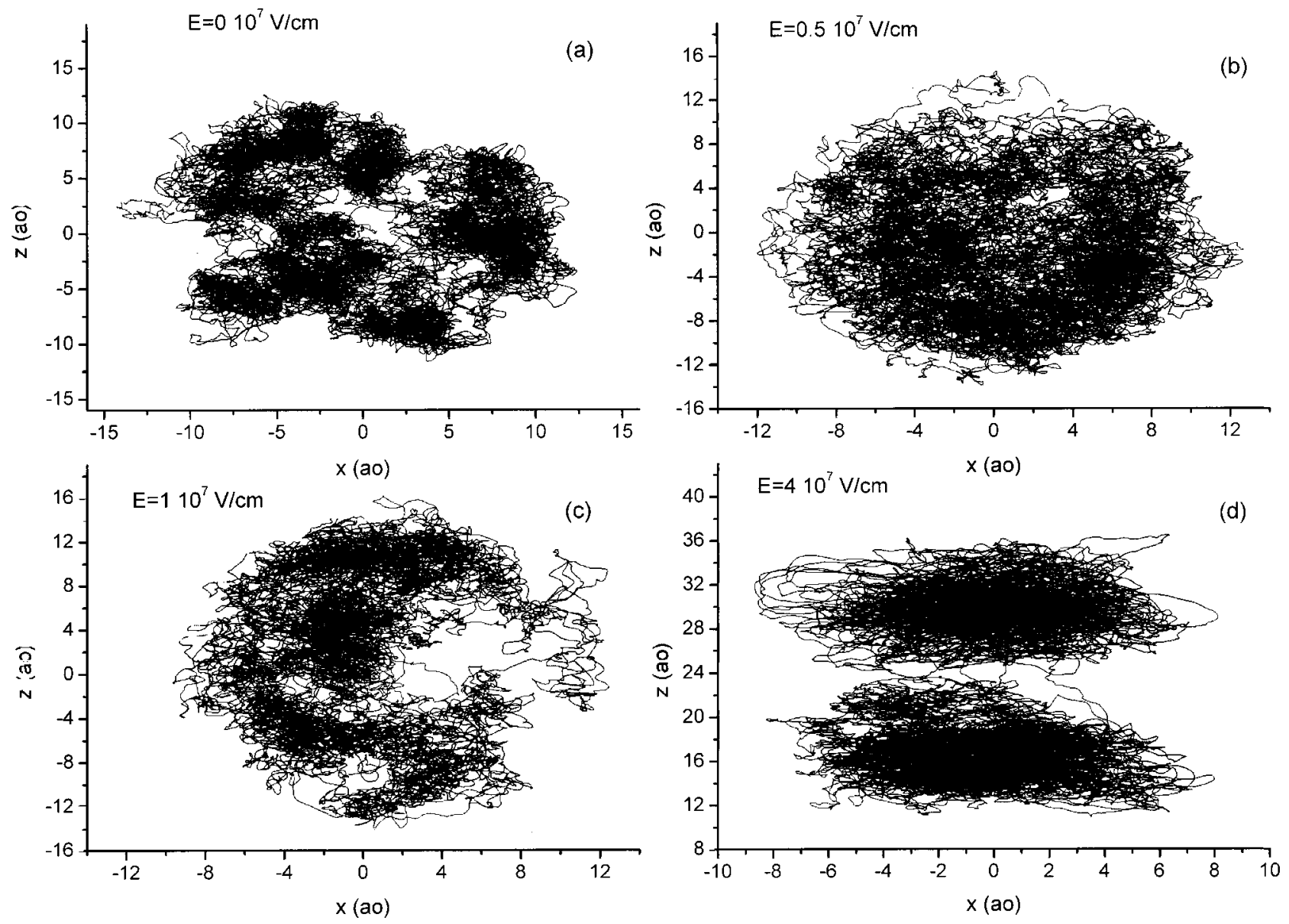

FIG. 2. Single particle trajectories projected onto the $x-z$ plane for (a) $E=0$, (b) $0.5 \times 10^{7} \mathrm{~V} / \mathrm{cm}$, (c) $1 \times 10^{7} \mathrm{~V} / \mathrm{cm}$, and (d) $4 \times 10^{7} \mathrm{~V} / \mathrm{cm}$.

\section{B. Self-van Hove correlation functions}

For a more detailed analysis of the spatial-temporal correlations of single particle motions with themselves, we calculated the self-part of the van Hove density autocorrelation function, which is defined as ${ }^{33}$

$$
G_{s}(r, t)=\frac{1}{N} \sum_{i}^{N}\left\langle\delta\left[r_{i}(t)-r_{i}(0)-r\right]\right\rangle .
$$

The function $4 \pi r^{2} G_{S}(r, t)$ represents the probability of finding a particle $i$ at $(r, t)$ given that the same particle was at the origin $r=0$ at $t=0$. The term $r$ is the radial distance of an oxygen atom from the origin which is taken at the center of mass of the cluster. These functions have the following asymptotic properties:

$$
\begin{aligned}
& G_{S}(r, 0)=\delta(r), \\
& G_{S}(r, t)=V^{-1},
\end{aligned}
$$

where the latter expression implies that the particle asymptotically can be found anywhere.

In the hydrodynamic limit, the $G_{S}(r, t)$ functions are described by the Gaussian propagator

$$
r^{2} G_{s}(r, t)=\frac{r^{2}}{(4 \pi D t)^{3 / 2}} e^{-r^{2} / 4 D t},
$$

where $D$ is the self-diffusion coefficient.
The $r^{2} G_{S}(r, t)$ functions for $E=0,0.5 \times 10^{7}, 2 \times 10^{7}$, $3 \times 10^{7}, 5 \times 10^{7}$, and $7 \times 10^{7} \mathrm{~V} / \mathrm{cm}$ are illustrated at specific times in Figs. 4(a)-4(f), respectively. For fields $E<E_{C 1}$ the curves exhibit a single peak which moves to larger distances with increasing time. At longer times the distributions become stationary, since, as seen from Fig. 1, the self-diffusion coefficient goes to zero. The curves are not perfect Gaussians, even in the long time limit and are centered at about 12 ao, which corresponds to the radial position of third neighbors, as estimated from the corresponding oxygen-oxygen pair correlation functions. ${ }^{17}$ Especially for $E=0.5 \times 10^{7}$ $\mathrm{V} / \mathrm{cm}$, stationary distributions are obtained at much earlier times than at zero field, as the result of the larger mobility of the particles. The same applies to $E=1 \times 10^{7} \mathrm{~V} / \mathrm{cm}$ as well.

However, at the transition field $E_{C 1}$ and for fields stronger than that, a change in the diffusion mechanism is observed, in a sense that at longer times the main peak becomes arrested at a position corresponding to second (first) neighbors depending on whether the field is weaker (stronger) than $E_{C 2}=5.0 \times 10^{7} \mathrm{~V} / \mathrm{cm}$, whereas a secondary shoulder builds up at larger distances. The appearance of shoulders signifies the onset of activated jump events, and this effect has been considered as the crossover from the liquid to the glassy state in a system of soft spheres. ${ }^{34,35}$ Pronounced shoulders are observed in Fig. 4(c) at $t=1.21 \mathrm{~ns}$ for $E=2 \times 10^{7} \mathrm{~V} / \mathrm{cm}$ and in Fig. 4(d) at $t=1.67 \mathrm{~ns}$ for $E=3 \times 10^{7} \mathrm{~V} / \mathrm{cm}$.

Continuous solid lines in Fig. 4 are fits to the asymptotic 

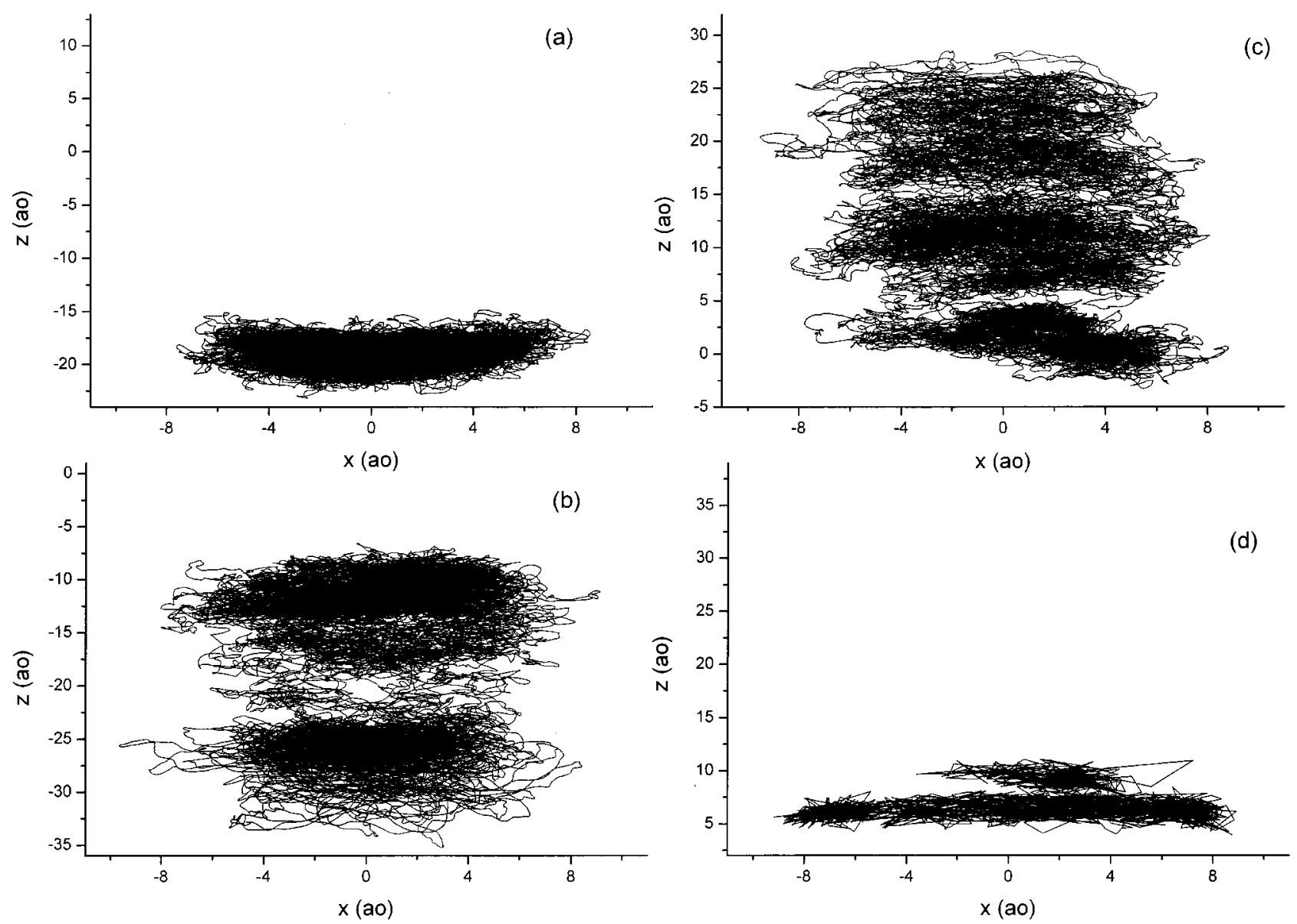

FIG. 3. Typical $1.5 \mathrm{~ns}$ single particle trajectories showing, (a) localization, (b) a single jump, (c) a double jump, and (d) jumps between locations on the $x$ - $y$ plane. Notice, that the $x$ and $z$ axes are of the same scale.

(long-time) approximation of the continuous time random walk probability distribution functions, pertinent to dispersive diffusion $(0<\delta<1) .{ }^{32,36}$ We have found that the computed $r^{2} G_{S}(r, t)$ curves can be fitted very well by the large $\xi$ approximation of the propagators, which have the following form:

$$
r^{2} P(r, t) \sim r^{2} b_{0} t^{-\delta d / 2} \xi^{\beta} e^{-b_{1} \xi^{v}},
$$

where

$$
\begin{aligned}
& \xi=d^{1 / 2} r / t^{\delta / 2}, \\
& v=2 /(2-\delta), \\
& \beta=d(\delta-1) /(2-\delta),
\end{aligned}
$$

and $d$ is the dimension of the Euclidian space.

In Fig. 5 we plot the position of the maximum peak of the $r^{2} G_{s}(r, t)$ probability distribution functions (PDFs) as a function of time. Since the transport is dispersive, the position corresponding to the maximum of the distribution should not be confused with the mean position. However, since the PDFs asymptotically are nearly Gaussians, (the dispersion parameter $\delta$ lies between 0.91 and 0.97 ), we can accept that this is approximately so. It is observed that the curves cluster in three distinct groups. One group, which is taken for fields $E<E_{C 1}$ gives asymptotically most probable displacements corresponding to third neighbors. The second group of curves is taken for fields $E_{C 1} \leqslant E<E_{C 2}$ and gives most probable displacements that correspond to second neighbors, whereas for fields stronger than these, diffusive particles hardly reach their first neighbors.

In investigations of the nature of transport in glassy systems and, in particular, in silicate glasses, Hiwatari and collaborators $^{37-40}$ showed that diffusion is facilitated not through single jump motions, but through forward correlated jumps, which require cooperative motions of two or more particles. In this kind of motion one particle occupies the position of another particle, which in turn moves simultaneously forward to another previously occupied site and so forth. Single jump motions have a large backward correlation probability and therefore do not promote diffusion.

Cooperative jumps of as many as five particles along the $z$ axis have been identified for $E=E_{C 2}$ as shown in Fig. 6(a). Also, a cooperative hopping event involving two particles for $E=7 \times 10^{7} \mathrm{~V} / \mathrm{cm}$ is displayed in Fig. 6(b). By inspecting several trajectories we came to the conclusion that diffusion for fields less than $E<E_{C 1}$ is mostly promoted by almost continuous-type of motion characterized by small waiting time intervals and large excursions traversing the entire length of the cluster. On the contrary, for fields equal or stronger than $E_{C 1}$, motion can be characterized as trapped, where diffusion is carried out through hopping events, a small number of them being cooperative for $E \geqslant E_{C 2}$. 

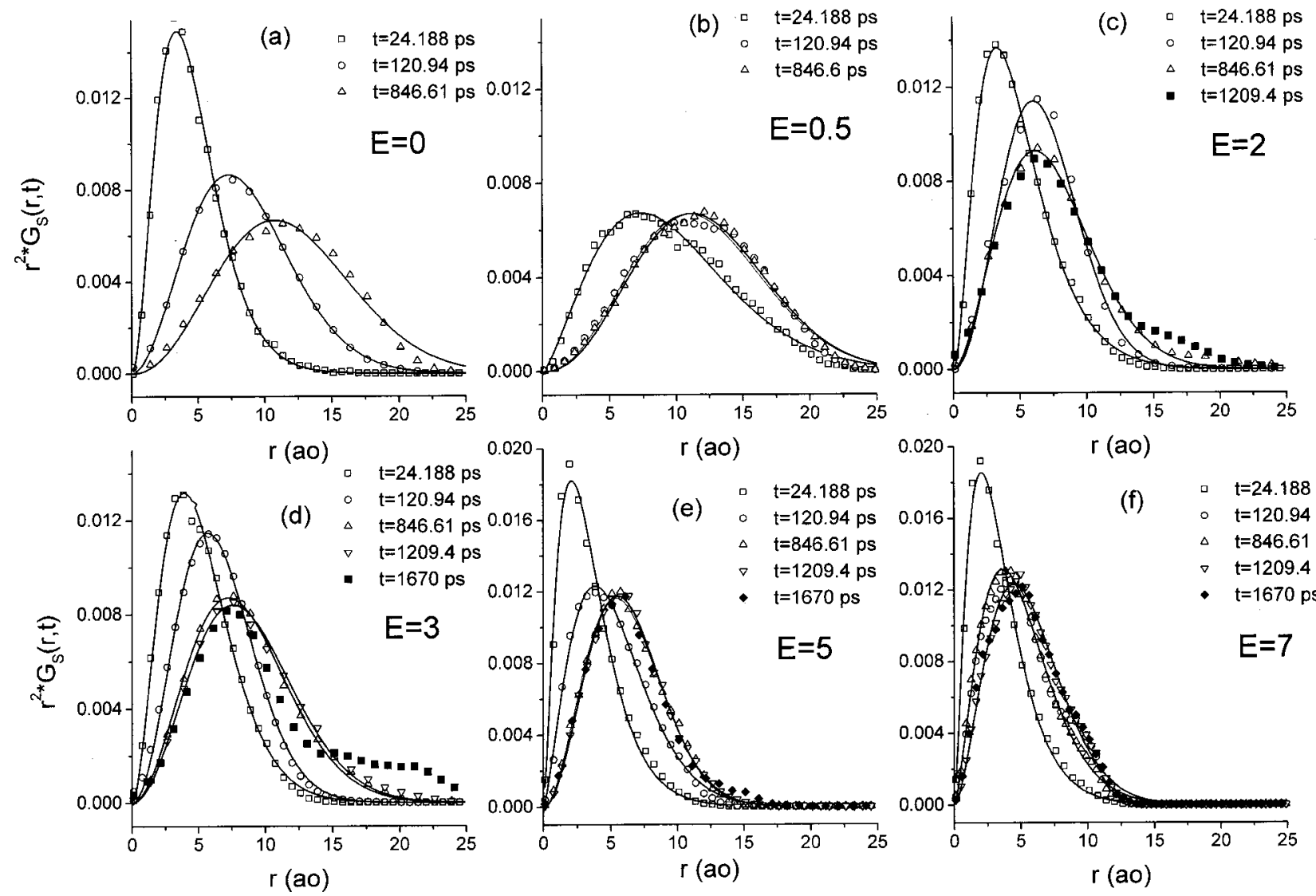

FIG. 4. The $r^{2} G_{s}(r, t)$ functions for (a) $E=0$, (b) $0.5 \times 10^{7} \mathrm{~V} / \mathrm{cm}$, (c) $2 \times 10^{7} \mathrm{~V} / \mathrm{cm}$, (d) $3 \times 10^{7} \mathrm{~V} / \mathrm{cm}$, (e) $5 \times 10^{7} \mathrm{~V} / \mathrm{cm}$, and (f) $7 \times 10^{7} \mathrm{~V} / \mathrm{cm}$. The corresponding times (in ps) are shown in each frame separately. Solid lines are fits to Eq. (13).

The slowing down of the structural relaxation is investigated in the following section, through the examination of the self-part of the intermediate scattering factor.

\section{Intermediate scattering function along field axis: Relaxation rates}

The self-part of the incoherent intermediate scattering function (ISF) is defined as

$$
F_{S}(Q, t)=\frac{1}{N} \sum_{i}^{N}\left\langle\exp \left\{i Q \cdot\left[r_{i}(t)-r_{i}(0)\right]\right\}\right\rangle
$$

and is the spatial Fourier transform of the self-part of the van Hove correlation function [Eq. (10)]. The term $r$ denotes the position of the oxygen atom. Because of the anisotropy of the system, we chose to calculate the ISFs along the $z$ direction only, for wave vectors $Q$ in the $1.1-3.6 \AA^{-1}$ range. All quantities derived from the analysis of the ISF curves will

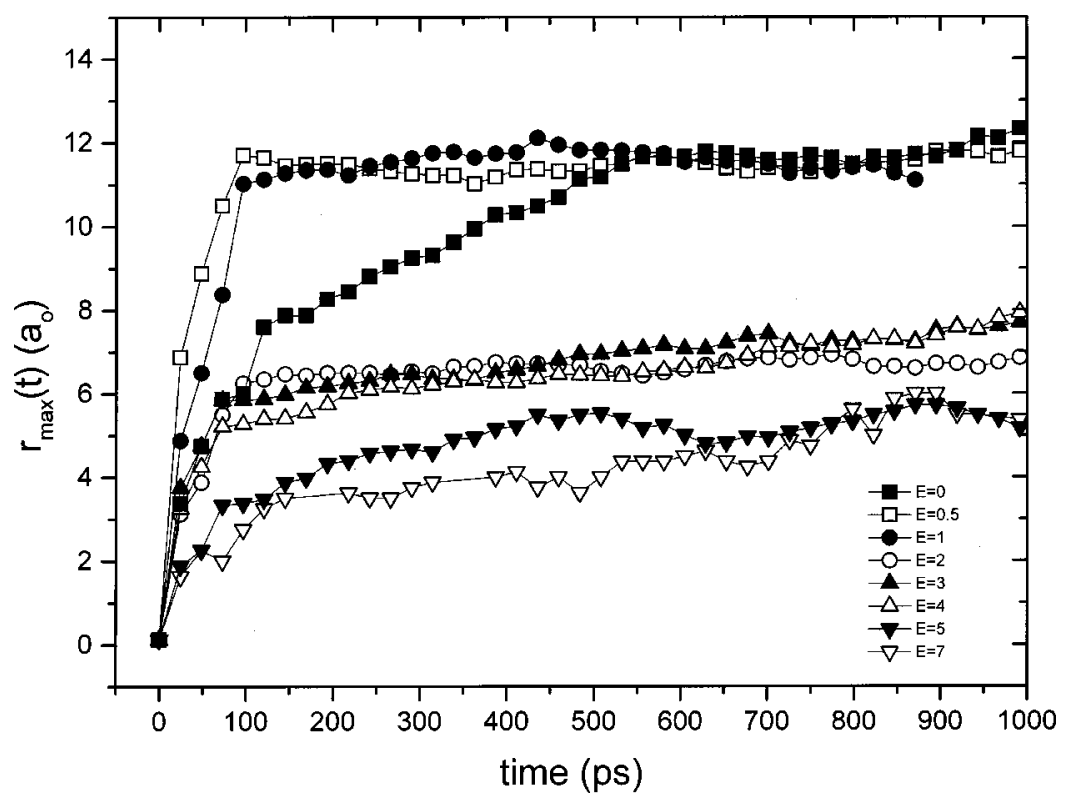

FIG. 5. The maximum of the $r^{2} G_{s}(r, t)$ distributions as a function of time. Fields are in $10^{7} \mathrm{~V} / \mathrm{cm}$. 


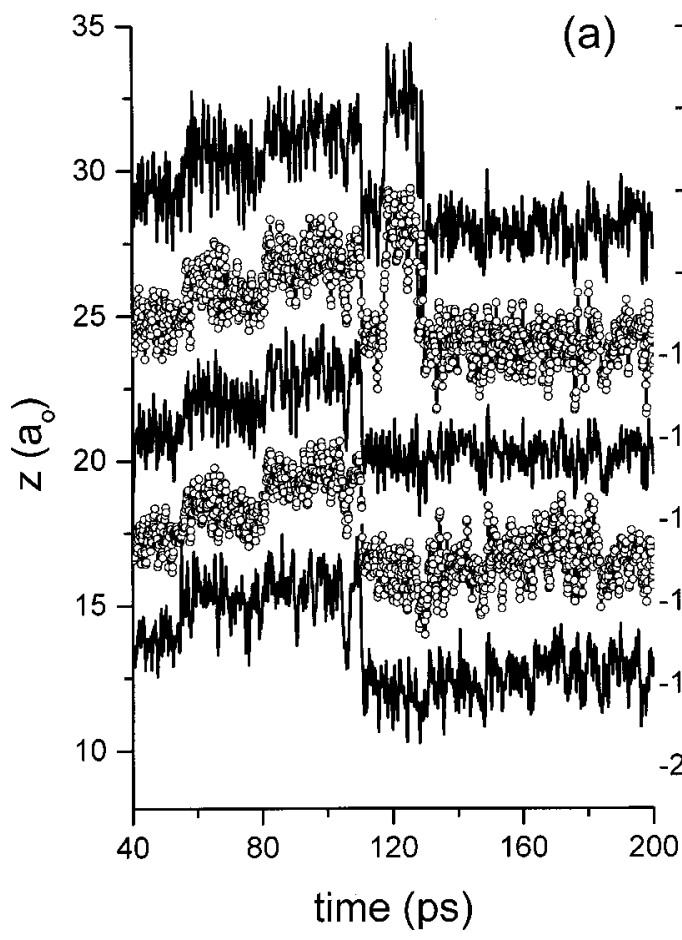

refer thereafter to relaxations along the direction of the external field.

The ISF curves for the particular wave vector $Q$ $=2.2 \AA^{-1}$, which corresponds to the main peak of the static structure factor of water, are presented in Fig. 7. For $E=0$ we observe a plateau, which, according to MCT is the signature for the existence of two different time scales in the relaxation process. The short-time part of the ISF corre- (b)

FIG. 6. Cooperative jump motions (a) of five particles $\left(E=5 \times 10^{7} \mathrm{~V} / \mathrm{cm}\right)$ and (b) of two particles $(E=7$ $\times 10^{7} \mathrm{~V} / \mathrm{cm}$ ), along the $z$ axis.

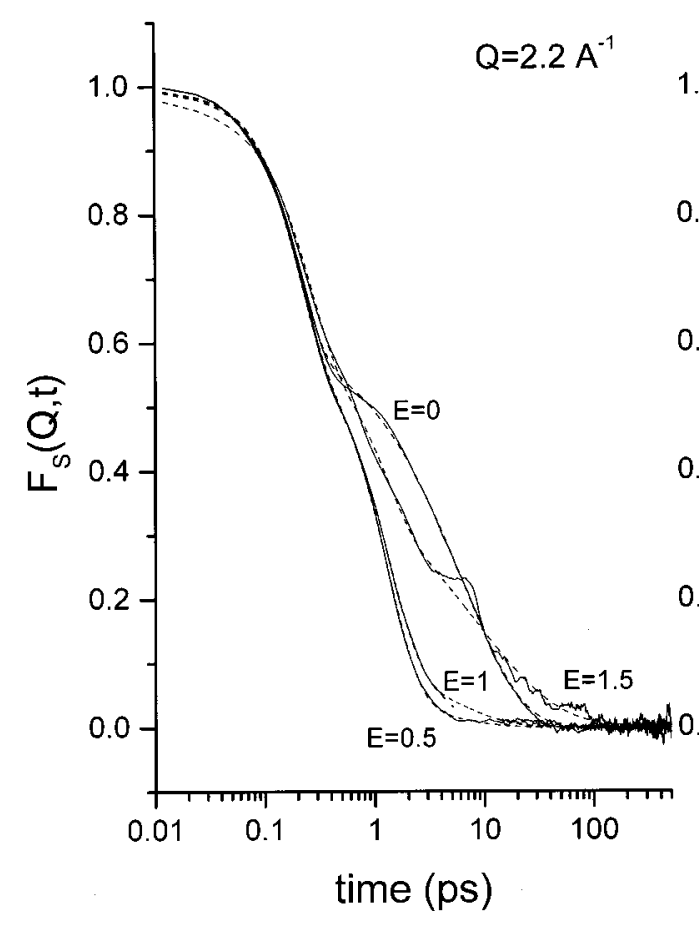

sponds to the relaxation of the motions inside the cage, whereas the long-time part to the cage relaxation ( $\alpha$ relaxation). The plateau, as in MSD, almost disappears with increasing field and for $E<E_{C 1}$, as it would happen in a system in the liquid state. As the field is further increased, the plateau starts gradually to build up again, whereas it reappears in a more pronounced way for $E \geqslant E_{C 2}$. These curves

are well fitted by the following functional form: ${ }^{24}$

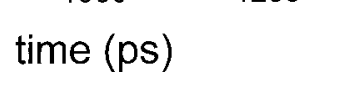



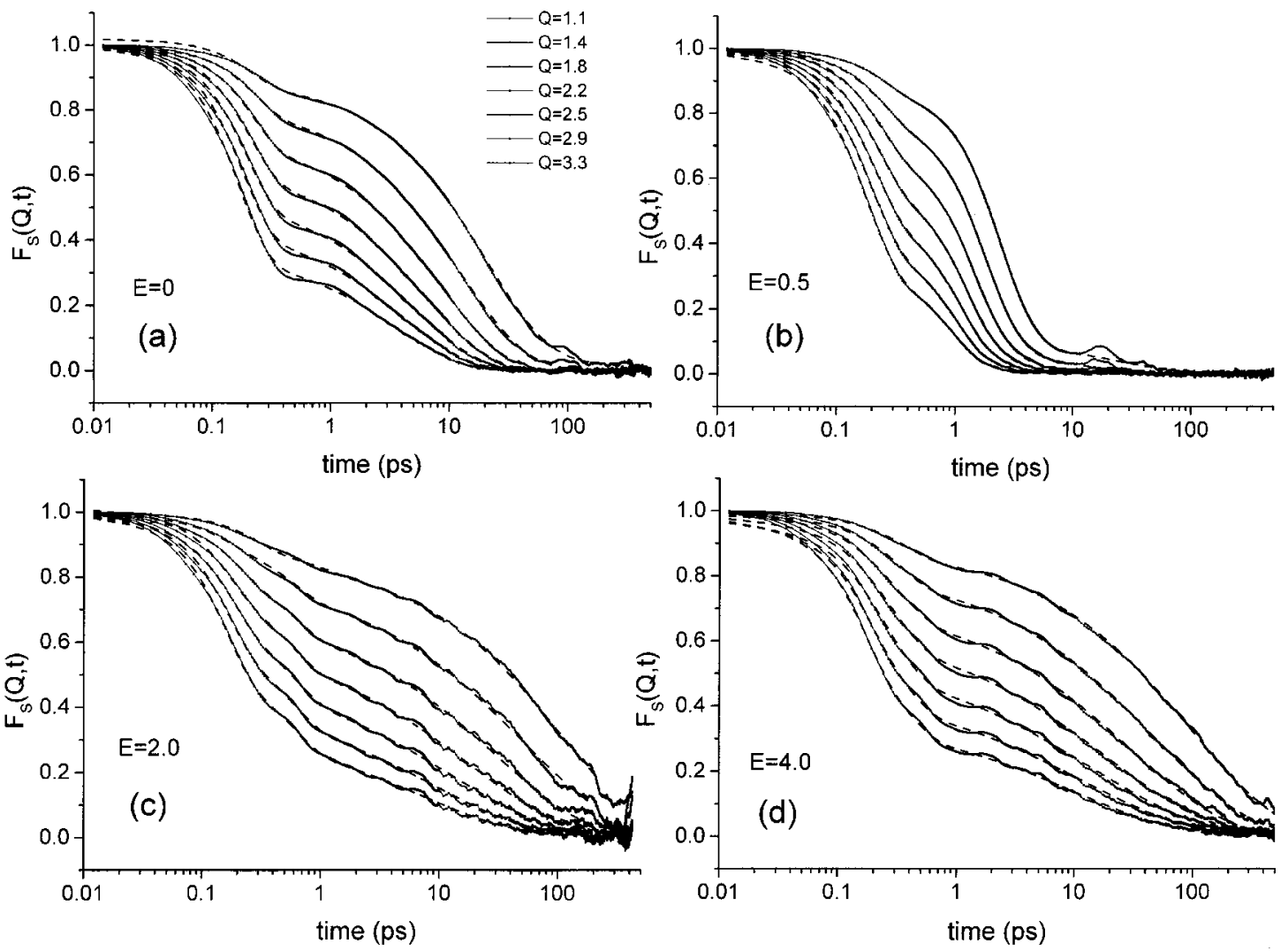

FIG. 8. Intermediate scattering functions (solid lines) calculated for $Q=1.1-3.3 \AA^{-1}$; (a) is for $E=0$, (b) is for $E=0.5 \times 10^{7} \mathrm{~V} / \mathrm{cm}$, (c) is for $E=2$ $\times 10^{7} \mathrm{~V} / \mathrm{cm}$, and (d) is for $4 \times 10^{7} \mathrm{~V} / \mathrm{cm}$. Dash lines are fits to Eq. (15).

$$
\begin{aligned}
F_{S}(Q, t)= & \exp \left\{-Q^{2} u_{0}^{2}\left[\frac{1-C}{\omega_{1}^{2}}\left(1-e^{\omega_{1}^{2} t^{2} / 2}\right)\right.\right. \\
& \left.\left.+\frac{C}{\omega_{2}^{2}}\left(1-e^{\omega_{2}^{2} t^{2} / 2}\right)\right]\right\} \exp \left(-\left(\frac{t}{\tau}\right)^{\beta}\right),
\end{aligned}
$$

where the first term in the product describes the short term behavior of the molecule inside the cage, whereas the second one is the usual Kohlrausch-Williams-Watts term for the description of the $\alpha$ relaxation process. $u_{0}, C, \omega_{1}, \psi_{2}, \beta$, and $\tau$ are fitting parameters which, in general, depend on $Q$. $\beta$ is the stretching exponent which varies between 0 and 1 and $\tau$ is the structural relaxation time. We found that it was not possible to reproduce well, both the short and long time behavior of the ISF curves with the commonly employed phenomenological expression

$$
\begin{aligned}
F_{S}(Q, t)= & {[1-A(Q)] \exp \left[-\left(\frac{t}{\tau_{s}}\right)^{2}\right] } \\
& +A(Q) \exp \left[-\left(\frac{t}{\tau_{1}}\right)^{\beta}\right],
\end{aligned}
$$

where $A(Q)$ is the Debye-Waller factor.

The quality of the fit is shown in Figs. 8(a)-8(d), where the $Q$ dependence of $F_{S}(Q, t)$ is displayed at four selected fields: (a) is for $E=0$, (b) is for $E=0.5 \times 10^{7} \mathrm{~V} / \mathrm{cm}$, (c) is for $E=2 \times 10^{7} \mathrm{~V} / \mathrm{cm}$, and (d) is for $E=4 \times 10^{7} \mathrm{~V} / \mathrm{cm}$. The dash lines in the semilogarithmic plots of Figs. 7 and 8 are the best fits to Eq. (15). The relaxation times $(\tau)$ and the Kohl- rausch exponents $(\beta)$ for $Q=2.2 \AA^{-1}$, as a function of the external field, are shown in Fig. 9. Notice that the relaxation times are presented in a logarithmic scale because of the large difference in their magnitude at weak and strong fields.

Consistent with our previous observations that for fields less than $E_{C 1}$ there is a loosening of the molecular cage, we observe a drop of the cage relaxation time to values smaller than that for $E=0$. For fields between $E_{C 1}$ and $E_{C 2}$ there is an abrupt increase of the relaxation times, which take almost the same values. Finally, for fields $E \geqslant E_{C 2}$ there is another jump in relaxation times, this time almost by an order of magnitude.

Figure 9 shows very clearly that the route to crystallization of the water cluster does not proceed in a single step, but rather involves clear-cut zones with different dynamic properties. Thus, we may infer that weak fields induce a transition from the solid to the liquid state. Next, for a broad range of fields, the system is found in a different state, which is neither liquid, nor crystalline. Also, this state has no resemblance to the state the cluster is found at $E=0$. This information comes not only from the present structural relaxation times but also from structural parameters calculated before in Ref. 17. And, finally, for strong enough fields the system is found in the crystalline state, as structural parameters and relaxation times show.

Similarly, the Kohlrausch exponent (in the inset of Fig. 9) shows an increase at $E=0.5 \times 10^{7} \mathrm{~V} / \mathrm{cm}$, which is consistent with the liquid-like behavior of the cluster at weak 


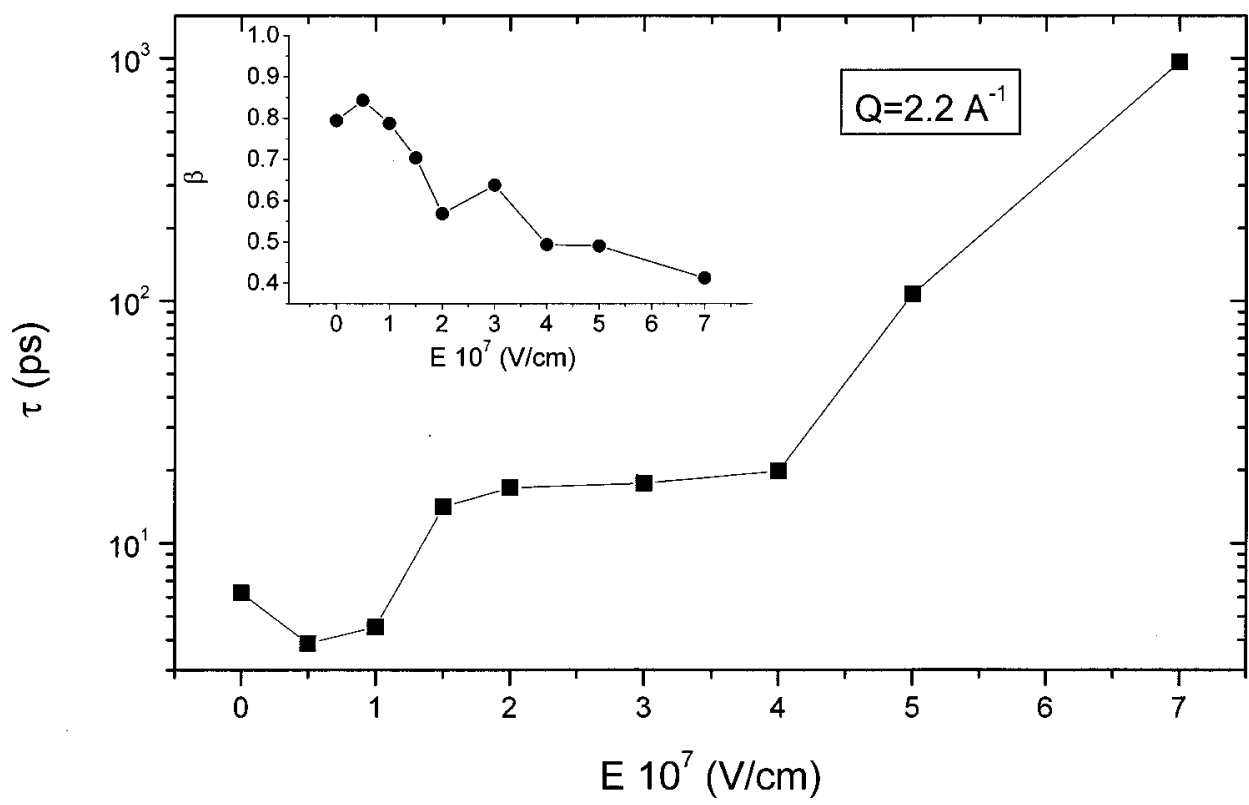

FIG. 9. Structure relaxation times (in logarithmic scale) as a function of the external field. The Kohlrausch exponent $(\beta)$ is shown in the inset. $Q$ $=2.2 \AA^{-1}$.

fields. Notice, that in the hydrodynamic limit $\beta$ becomes equal to 1 . With further field increase the exponent drops progressively to a value $\beta=0.4$ at $E=7 \times 10^{7} \mathrm{~V} / \mathrm{cm}$. A similar decrease of the exponent $\beta$ has also been observed experimentally during the isothermal crystallization process of a glassy polymer. ${ }^{41}$ According to the coupling model introduced by Ngai and León ${ }^{42,43}$ and references therein, which assigns a physical significance to the Kohlrausch exponent, by relating it to the existence of cooperative phenomena, ${ }^{42,43}$ the decrease of $\beta$ here can be understood as the increase of cooperativity requirements for the relaxation of the molecular units, because of the restrictions imposed by crystallinity.

In the following, we examine the dependence of the structural relaxation times on the wave vector $Q$ in the
[1.1-4 $\left.\AA^{-1}\right]$ range. Relaxation rates $(1 / \tau)$ as a function of $Q$, at several fields are presented in Fig. 10 in a $\log -\log$ plot. Solid lines are fits to the power-law function

$$
\frac{1}{\tau} \approx Q^{\gamma}
$$

Two general observations are made. First, the relaxation rates as a function of $Q$ cluster together within a particular range of external fields, namely for $E<E_{C 1}$ and $E_{C 1} \leqslant E$ $<E_{C 2}$. Therefore, the states that are found between $E_{C 1}$ and $E_{C 2}$ have similar relaxation rates not only for $Q=2.2 \AA^{-1}$, but also for a wider range of $Q$. Second, relaxation rates obey the power law of Eq. (17) fairly well, although the exponent

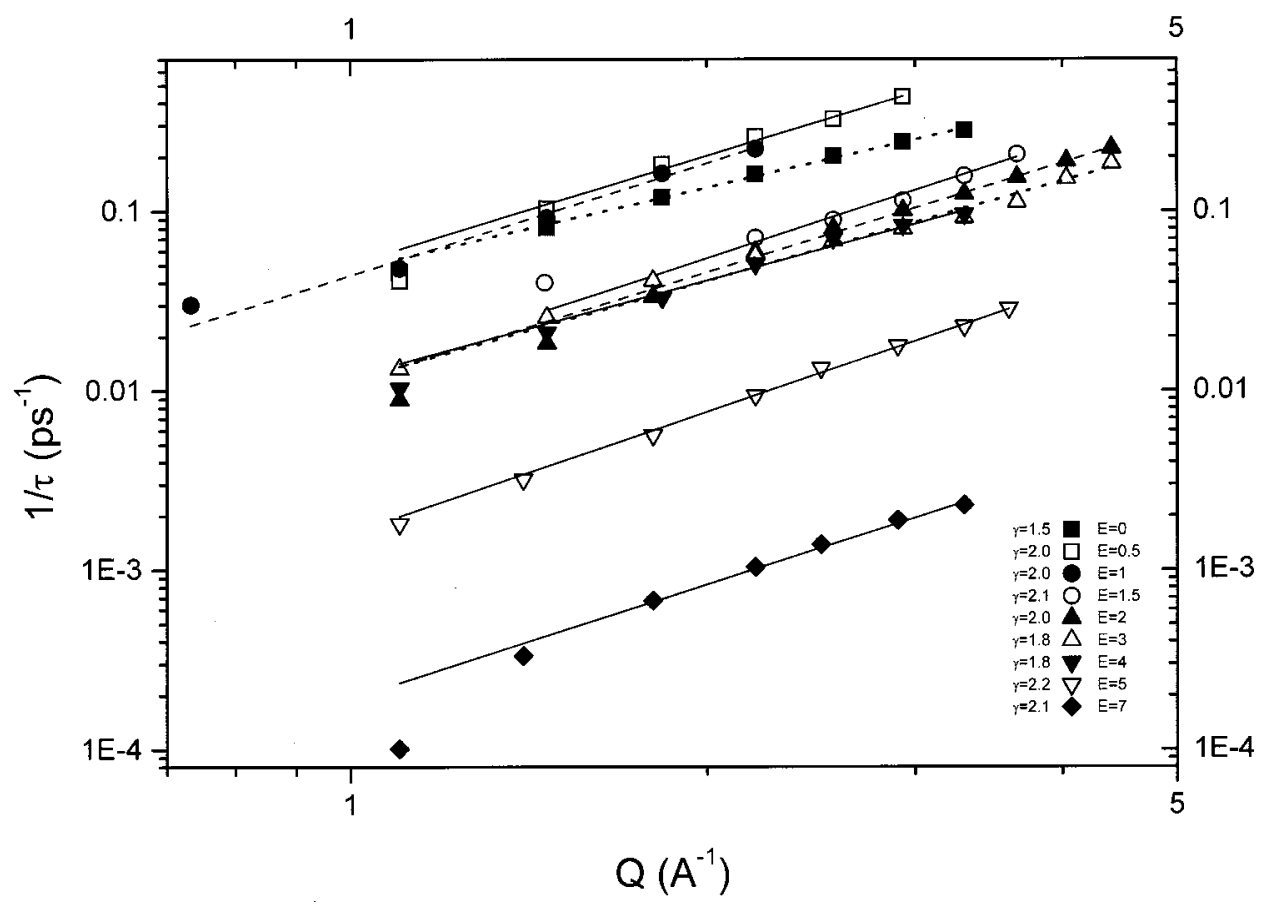

FIG. 10. $Q$ dependence of the relaxation rates $(1 / \tau)$ for several fields in a $\log -\log$ plot. Straight lines are fits to a power law, $\sim Q^{\gamma}$. The exponents are displayed in the figure. 


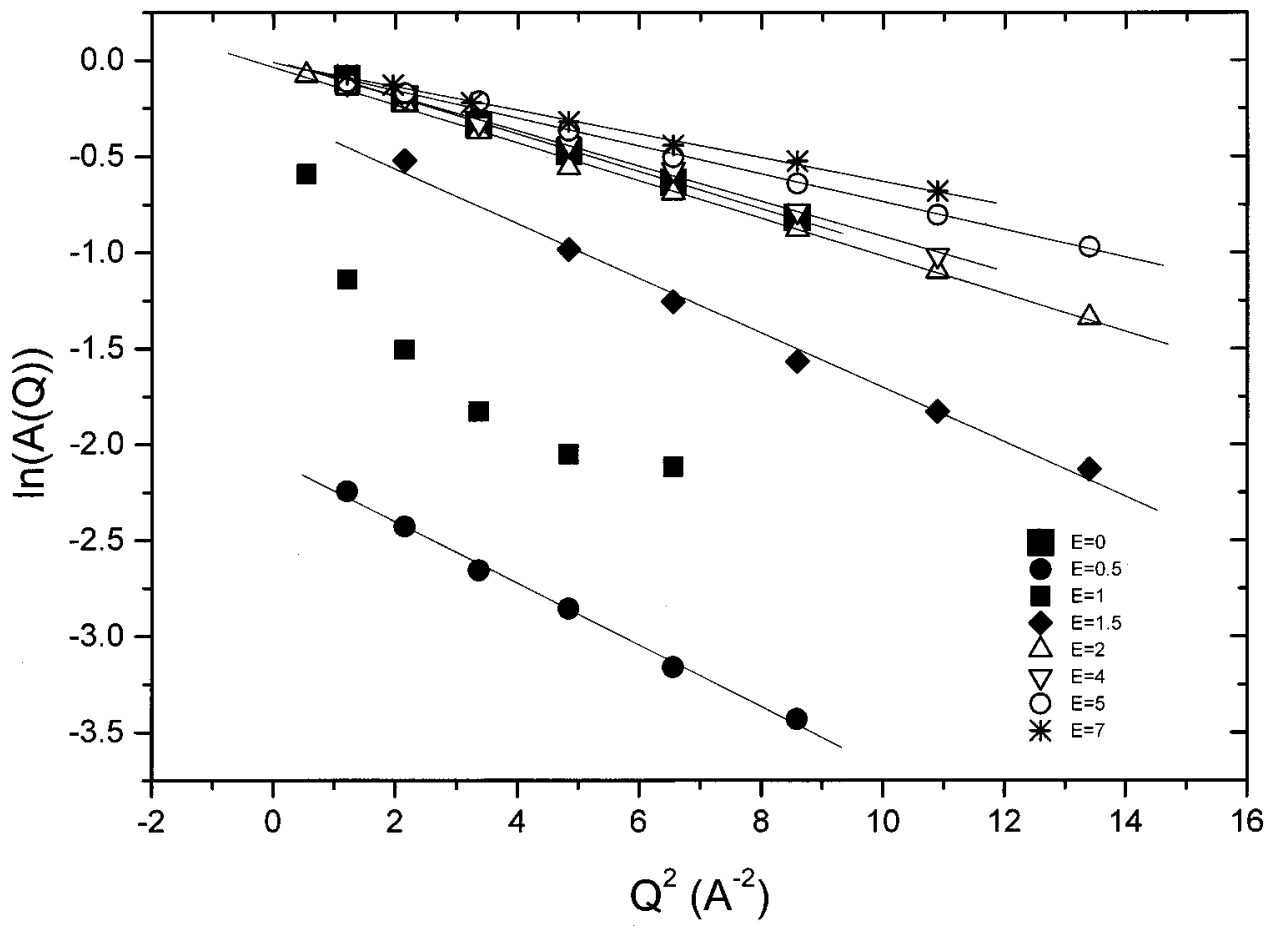

FIG. 11. The logarithm of the DebyeWaller factor as a function of $Q^{2}$. Straight lines are fits to Eq. (18). $\gamma$ depends on the range of wave vectors included in the fitting. The values of the exponents for each electric field, estimated from all $Q$ between 1.1 and $3.3 \AA^{-1}$, are displayed in Fig. 10 .

In general, for a bulk system obeying Gaussian dynamics in the small $Q$ limit, namely in distances larger than the molecular cage, $\gamma$ should be equal to 2 . However, with decreasing temperature, the $Q$ dependence of the relaxation rate was found to change gradually from quadratic $(\gamma=2)$ to linear $(\gamma=1)$, as simulations ${ }^{21}$ and experiments ${ }^{44}$ in supercooled water have shown. This result is reproduced here for $E=0$ where $\gamma$ is equal to 1.5 . The fact that $\gamma$ equals 2 for $E=0.5 \times 10^{7}$ and $E=1.0 \times 10^{7} \mathrm{~V} / \mathrm{cm}$ is consistent with the liquid-like picture of the cluster we have established for fields larger than zero and less than $E_{C 1}$.

However, it is surprising that $\gamma$ is not far from 2 for the strongest fields as well, for which a considerable amount of trapping along the $z$ direction is found and where the motion is far from being of the Debye type. We have found that for these fields, the Kohlrausch exponent $(\beta)$ never reaches the value 1 (as it should be in normal diffusion), even for the smallest wave vector examined here $\left(0.73 \AA^{-1}\right)$. Instead, for all $E \geqslant E_{C 1}$ it varies between 0.6 and 0.4 as $Q$ is varied. This kind of behavior is characteristic of subdiffusive motion and it was also observed in glass-forming polymers. ${ }^{45}$

In general $\gamma$ is related to the fractal dimension of the space where the system moves. ${ }^{46}$ When, the long-range motion of the system becomes restricted due to large connectivity, then $\gamma>2$. Here, this rule is marginally obeyed for $E$ $\geqslant E_{C 2}$, where indeed the cluster structure is close to crystalline. Here, $\gamma=2.2$ and 2.1 for $E=5 \times 10^{7}$ and $E=7$ $\times 10^{7} \mathrm{~V} / \mathrm{cm}$, respectively. The same also holds true for $E$ $=E_{C 1}(\gamma=2.1)$, where the structure, as characterized by a number of stacked cubes, displays some kind of order. Therefore, the values of $\gamma$ we get for these fields should not be accidental. On the other hand, it was suggested ${ }^{46}$ that an exponent $\gamma$ less than 2 is related to features of the jump path and to enhanced dynamics. The fact that $\gamma \leqslant 2$ in the $E_{C 1}$ $<E<E_{C 2}$ range is an encouraging result, towards the correct direction. However, the way this exponent is related to the exact nature of the disorder is the scope of another work.

According to Ref. 24, the incoherent Debye-Waller factor is defined as

$$
A(Q)=\exp \left[-Q^{2} u_{o}^{2}\left(\frac{1-C}{\omega_{1}^{2}}+\frac{C}{\omega_{2}^{2}}\right)\right]
$$

which has a Gaussian dependence on $Q$. Alternatively, the Debye-Waller factor can be written as

$$
A(Q)=\exp \left(-Q^{2} \alpha^{2} / 3\right)
$$

where $\alpha$ represents the mean vibrational amplitude of water molecules in a spherical cage. The logarithm of $A(Q)$ as a function of $Q^{2}$ is displayed in Fig. 11 for several fields. Straight lines are fits to $\ln A(Q)$. As we can see there is a very good linear dependence, except for $E=1 \times 10^{7} \mathrm{~V} / \mathrm{cm}$. The values of $\alpha$ which are determined from the slope of the corresponding lines, as a function of $E$, are illustrated in Fig. 12. In this case, in order to compare $\alpha$ with values from literature, we assume that the vibrational amplitude is isotropic in all directions. We can clearly see that there is an increase of the vibrational amplitude inside the cage for $0<E<E_{C 1}$, whereas for further field increase $\alpha$ decreases monotonically. For $E=0, \alpha$ is equal to $0.53 \AA$, which is comparable to the corresponding experimental value of supercooled water, which for a wide range of temperatures was found equal to $0.48 \AA{ }^{28}$ For $E=7 \times 10^{7} \mathrm{~V} / \mathrm{cm}, \alpha$ drops to $0.43 \AA$. The variation of $\alpha$ with $E$ is consistent with the main findings of this work. 


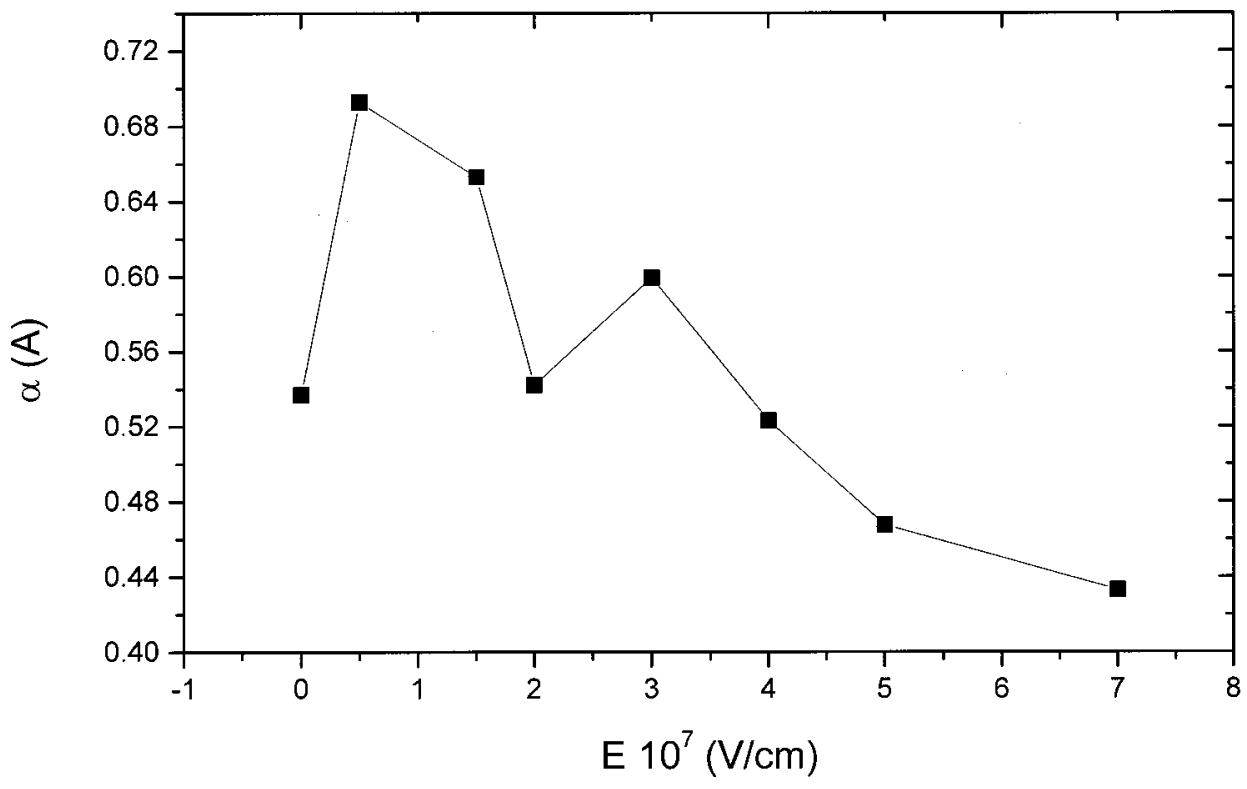

FIG. 12. The mean vibrational amplitude $(\alpha)$ of a water molecule as a function of the external field.

\section{SUMMARY AND CONCLUSIONS}

In this work, single particle translational dynamics of a cold $(T=200 \mathrm{~K})$, water cluster are investigated in detail as a function of an external uniform, electric field. In a previous work, ${ }^{16,17}$ structural transformations and molecular axis reorientational dynamics had been examined. Here, we mainly concentrate on the dynamical behavior along the field axis $(z$ axis), due to cluster elongation induced after a particular field strength, $\left(E_{C 1}\right)$. In directions perpendicular to the field, $\left(x-y\right.$ plane), water molecules have a single neighbor only, ${ }^{17}$ therefore any notion to dynamics is rather meaningless. Because of this, mean-square-displacement curves and intermediate scattering functions calculated on the $x-y$ plane have been found to be identical for $E \geqslant E_{C 1}$, showing no variations with field increase.

Our main results can be summarized as follows: (i) Two critical fields $\left(E_{C 1}\right.$ and $\left.E_{C 2}\right)$ for which the cluster behavior is drastically changed, have been identified. $E_{C 1}$, which is equal to $1.5 \times 10^{7} \mathrm{~V} / \mathrm{cm}$, is associated ${ }^{17}$ with the flipping of the molecular dipoles from random directions to directions restricted within a solid angle between 0 and $\pi / 2$ with respect to the field. $E_{C 2}$, which is equal to $5.0 \times 10^{7} \mathrm{~V} / \mathrm{cm}$, is associated with the transition to the crystal structure. The identification of these two fields was based unambiguously on the calculation of the structure relaxation times, which were found to change by two or three times at $E_{C 1}$ with respect to weaker fields, and to jump by almost ten times at $E_{C 2}$. (ii) The relaxation of the structure was described by a stretched exponential. Also, the $Q$ dependence of the relaxation rates for all fields was found to obey a power law with exponents varying in the [1.6-2.2] range. (iii) Weak fields, $0<E<E_{C 1}$, induce a significant loosening of the molecular cage. This result is extracted in a consistent way from the behavior of the MSD- $z$ curves, the self-part of the van Hove correlation functions, from the structure relaxation times $(\tau)$, the Kohlraush exponents $(\beta)$, and the mean amplitude of the in-cage vibrational motions $(\alpha)$. Specifically, the plateau present in the MSD- $z$ curves for $E=0$, disappears for these fields indicating a transition to a liquid-like state. Also, the van Hove functions obtain their asymptotic distributions at much earlier times than at $E=0$ and $E \geqslant E_{C 1}$. Furthermore, relaxation times decrease and the corresponding Kohlrausch exponents increase to values closer to one. Finally, the mean vibrational amplitude of the molecules increases considerably in this field region. The loosening of the molecular cage was also implied in our previous work, ${ }^{16,17}$ where a reduction of the reorientational rates of the molecular axes was observed.

\section{ACKNOWLEDGMENTS}

Financial support by a grant from INTAS (99-01162) is gratefully acknowledged. Also, Professor Wolffram Schröer and Dr. Cristos Varsamis are acknowledged for stimulating and helpful discussions.

${ }^{1}$ Y. H. Hwang and X.-I. Wu, Phys. Rev. Lett. 74, 2284 (1995).

${ }^{2}$ M. Kiselev and K. Heinzinger, J. Chem. Phys. 105, 650 (1996).

${ }^{3}$ I. M. Svishchev and P. G. Kusalik, Phys. Rev. Lett. 73, 975 (1994).

${ }^{4}$ I. M. Svishchev and T. M. Hayward, J. Chem. Phys. 111, 9034 (1999).

${ }^{5}$ I. M. Svishchev and P. G. Kusalik, J. Am. Chem. Soc. 118, 649 (1996).

${ }^{6}$ I. M. Svishchev and P. G. Kusalik, Phys. Rev. B 53, 8815 (1996).

${ }^{7}$ I. C. Yeh and M. L. Berkowitz, J. Chem. Phys. 110, 7935 (1999).

${ }^{8}$ X. Xia and M. L. Berkowitz, Phys. Rev. Lett. 74, 3193 (1995).

${ }^{9}$ D. H. Jung, J. H. Yang, and M. S. Jhon, Chem. Phys. 244, 331 (1999).

${ }^{10}$ R. O. Watts, Chem. Phys. 57, 185 (1981).

${ }^{11}$ S. B. Zhu and G. W. Robinson, J. Chem. Phys. 94, 1403 (1991).

${ }^{12}$ M. Watanabe, A. Brodsky, and W. P. Reinhardt, J. Chem. Phys. 95, 4593 (1991).

${ }^{13}$ H. E. Alper and R. M. Levy, J. Phys. Chem. 94, 8401 (1990).

${ }^{14}$ S. Senapati and A. Chandra, J. Mol. Struct.: THEOCHEM 455, 1 (1998).

${ }^{15}$ S. H. Lee, J. C. Rasaiah, and J. B. Hubbard, J. Chem. Phys. 86, 2383 (1987).

${ }^{16}$ S. V. Shevkunov and A. Vegiri, J. Mol. Struct.: THEOCHEM 574, 27 (2001).

${ }^{17}$ A. Vegiri and S. V. Shevkunov, J. Chem. Phys. 115, 4175 (2001).

${ }^{18}$ R. Tao, Phys. Rev. E 47, 423 (1993).

${ }^{19}$ J. M. Sun and R. Tao, Phys. Rev. E 52, 813 (1995).

${ }^{20}$ J. J. Ullo, Phys. Rev. A 36, 816 (1987).

${ }^{21}$ S.-H. Chen, P. Gallo, F. Sciortino, and P. Tartaglia, Phys. Rev. E 56, 4231 (1997). 
${ }^{22}$ P. Gallo, F. Sciortino, and P. Tartaglia, Phys. Rev. Lett. 76, 2730 (1996).

${ }^{23}$ F. Sciortino, P. Gallo, P. Tartaglia, and S.-H. Chen, Phys. Rev. E 54, 6331 (1996).

${ }^{24}$ S.-H. Chen, C. Liao, F. Sciortino, P. Gallo, and P. Tartaglia, Phys. Rev. E 59, 6708 (1999).

${ }^{25}$ C. Y. Liao, F. Sciortino, and S.-H. Chen, Phys. Rev. E 60, 6776 (1999).

${ }^{26}$ M. C. Bellissent-Funel, S. Longeville, J. M. Zanotti, and S.-H. Chen, Phys. Rev. Lett. 85, 3644 (2000).

${ }^{27}$ S.-H. Chen, J. Teixeira, and R. Nicklow, Phys. Rev. A 26, 3477 (1982).

${ }^{28}$ J. Teixeira, M.-C. Bellissent-Funel, S.-H. Chen, and A. J. Dianoux, Phys. Rev. A 31, 1913 (1985).

${ }^{29}$ W. Götze and L. Sjögren, Rep. Prog. Phys. 55, 241 (1992).

${ }^{30}$ W. L. Jorgensen, J. Chandrasekhar, J. D. Madura, R. W. Impey, and M. L. Klein, J. Chem. Phys. 79, 926 (1983).

${ }^{31}$ R. Metzler, E. Barkai, and J. Klafter, Phys. Rev. Lett. 82, 3563 (1998).

${ }^{32}$ J. Klafter, G. Zumofen, and A. Blumen, Chem. Phys. 177, 821 (1993).

${ }^{33}$ J. P. Hansen and I. R. McDonald, Theory of Simple Liquids, 2nd ed. (Academic, London, 1987).
${ }^{34}$ J. N. Roux, J. L. Barrat, and J. P. Hansen, J. Phys.: Condens. Matter 1, 7171 (1989)

${ }^{35}$ J. L. Barrat, J. N. Roux, and J. P. Hansen, Chem. Phys. 149, 197 (1990).

${ }^{36}$ J. Klafter and G. Zumofen, J. Phys. Chem. 98, 7366 (1994).

${ }^{37}$ H. Miyagawa, Y. Hiwatari, B. Bernu, and J. P. Hansen, J. Chem. Phys. 88, 3879 (1988).

${ }^{38}$ J. Habasaki, I. Okada, and Y. Hiwatari, Phys. Rev. B 55, 6309 (1996)

${ }^{39}$ J. Habasaki and Y. Hiwatari, Phys. Rev. E 59, 6962 (1998).

${ }^{40}$ Y. Hiwatari and J. Habasaki, J. Phys.: Condens. Matter 12, 6405 (2000).

${ }^{41}$ T. A. Ezquerra, J. Majszczyk, F. J. Baltà-Calleja, E. López-Cabarcos, K. H. Gardner, and B. S. Hsiao, Phys. Rev. B 50, 6023 (1994).

${ }^{42}$ K. L. Ngai, J. Chem. Phys. 111, 3639 (1999).

${ }^{43}$ C. León and K. L. Ngai, J. Phys. Chem. B 103, 4045 (1999).

${ }^{44}$ J. M. Zanotti, M. C. Bellissent-Funel, and S. H. Chen, Phys. Rev. E 59, 3084 (1999).

${ }^{45}$ A. van Zon and S. W. de Leeuw, Phys. Rev. E 60, 6942 (1999).

${ }^{46}$ J. Habasaki, I. Okada, and Y. Hiwatari, Phys. Rev. E 52, 2681 (1995). 